\title{
Hybrid processing of SCADA and synchronized phasor measurements for tracking network state
}

\author{
Boris A. Alcaide-Moreno ${ }^{a}$ \\ balcaide@dep.fie.umich.mx
}

\author{
Claudio R. Fuerte-Esquivel ${ }^{a}$ \\ cfuerte@umich.mx
}

\author{
Mevludin Glavic $^{b}$ \\ mevludin.glavic@ulg.ac.be
}

\author{
Thierry Van Cutsem ${ }^{c}$ \\ t.vancutsem@ulg.ac.be
}

\begin{abstract}
This paper proposes a new tracking state estimator aimed at following some of the dynamics of the network state (bus voltage phasors) by a hybrid processing of SCADA and synchronized phasor measurements. The latter are assumed to be available in limited number. To avoid time skew effects, only the SCADA measurements received since the last execution of the estimator are processed. To ensure observability, estimated SCADA measurements are used as pseudo-measurements. The procedure includes a prediction, an innovation analysis and a correction step. The latter consists of solving a constrained least-squares optimization. The simulation results refer to a test system undergoing large disturbances, evolving to longterm voltage instability or stabilized by emergency control. The proposed method appears to satisfactorily track the overall network evolution, even during those severe conditions.
\end{abstract}

Index Terms-State estimation, tracking, synchronized phasor measurements, SCADA, constrained least-squares.

\section{INTRODUCTION}

The availability of synchronized phasor measurements has opened an avenue of new applications for monitoring and control of power systems [1], [2].

The approach presented in this paper is an extension of the one proposed in [3]. Its overall objective is to track the changing network state, i.e. the vector of complex bus voltages. The idea of Tracking State Estimation (TSE) is not new; it can be traced back to early works, e.g. [4], [5], [6]. However, the availability of synchronized phasor measurements allows envisaging a TSE executed at a much higher rate than stateof-the-art state estimation. The period of execution $T_{r}$ could be in the order of one second. Tracking the system evolution at this rate could bring new useful information in the control center, and increase system situational awareness.

In this work, only a limited number of PMUs are assumed to be available. This situation is expected to prevail for some time, in particular because a wide PMU deployment must be supported by adequate upgrades of communication infrastructures. Consequently, existing and near-future PMU configurations are far from ensuring full network observability, and methods are needed to exploit both SCADA and synchrophasor measurements. While the former are received in

a Faculty of Electrical Engineering, Universidad Michoacana de San Nicolás de Hidalgo, México. This work was in part supported by CONACyT, México under grant 321846.

${ }^{b}$ Dept. of Electrical Engineering and Computer Science, University of Liège, Belgium.

${ }^{c}$ Fund for Scientific research at the Dept. of Electrical Engineering and Computer Science, University of Liège, Belgium.

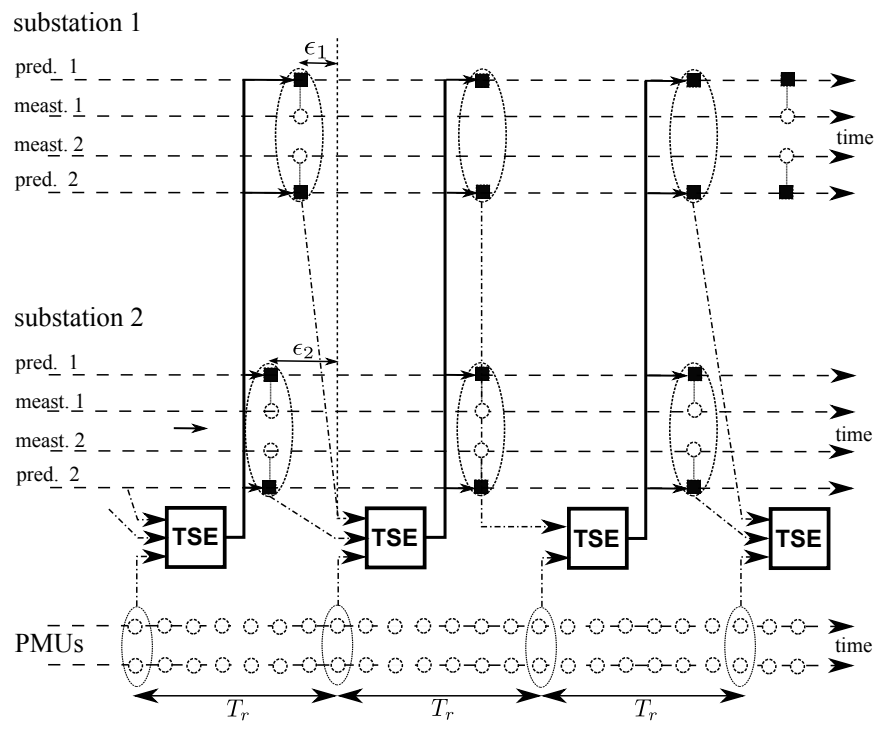

Figure 1. Use of SCADA, PMU and predicted SCADA measurements in the proposed TSE. Circles (o) indicate when a particular measurement is received by the SCADA system. Squares ( $\mathbf{a})$ denote predicted measurement values

the control center every two to five seconds, the latter are available at the rate of several tens of samples per second [7]. Methods are needed to exploit those asynchronously available real-time data in the best possible way [3], [8]. The approach of this paper exploits: (i) synchrophasor measurements in a systematic manner, (ii) SCADA measurements as and when they are received by the control center, along with (iii) recurrently predicted SCADA measurements ensuring observability.

\section{PRINCIPLE OF THE METHOD}

The principle of the proposed Tracking State Estimation (TSE) is shown graphically in Fig. 1 and can be summarized as follows.

At a given time $t$, the processed measurements consist of the most recent synchronized (bus voltage and branch current) phasor measurements as well as the new SCADA (voltage magnitude, active or reactive power) measurements that have been received since the last TSE execution, i.e. in the time interval $\left[t-T_{r}, t\right]$. SCADA measurements used at time $t$ are affected by delays $\delta_{j i}+\tau_{i}+\epsilon_{i}$ where $\delta_{j i}$ is a value between zero and $\Delta_{i}$, the period of the cyclic measurement gathering in the corresponding $i$-th substation, $\tau_{i}$ is the time for the set of data 


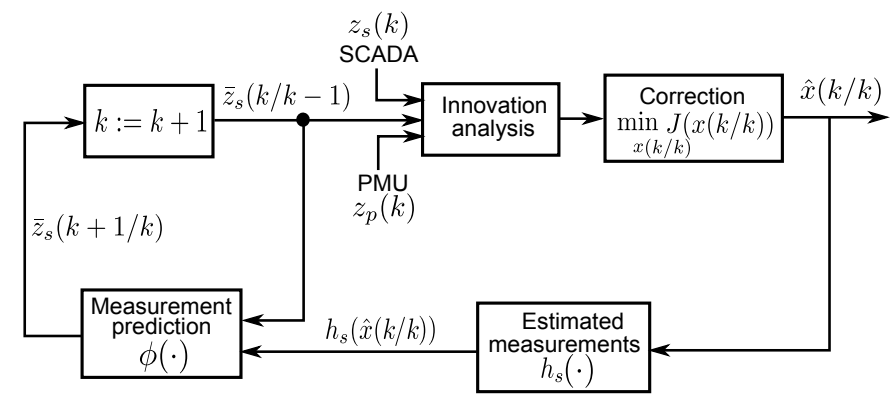

Figure 2. Main steps of the proposed tracking state estimation

to be received by the control center, and $\epsilon_{i}$ is the dead time between the arrival of measurements and their processing.

It is well known that standard state estimators suffer from time skew of their measurements, especially when the system is undergoing some dynamic evolution. In order to minimize this effect, SCADA measurements received before time $t-T_{r}$ are no longer used. This leads to using only a fraction of the whole set of SCADA measurements in each TSE.

Since they are both in limited number, SCADA and synchrophasor measurements make the system unobservable, and pseudo-measurements must be added to restore observability. The latter are obtained from the most recent TSEs, by predicting the values of all SCADA measurements, according to some time series analysis. These pseudo-measurements are referred to as predicted SCADA measurements.

At time $t$ the available SCADA, the synchronized phasor and the predicted SCADA measurements are processed all together in the least-square sense to obtain a new estimate of the state vector, from which the estimated values of all quantities measured by the SCADA system are obtained. The latter are used to predict the values of all SCADA measurements at time $t+T_{r}$.

Zero bus injections are also included in the formulation as constraints to improve the accuracy of the proposed method.

\section{MATHEMATICAL FORMULATION}

The proposed TSE method is outlined in Fig. 2. It consists of the following three major steps: measurement prediction, innovation analysis and correction.

\section{A. Measurement model}

Let $m$ be the total number of SCADA measurements. The measurement model is made up of the following equations:

$$
\begin{aligned}
\boldsymbol{z}_{s}(k) & =\boldsymbol{D}_{k} \boldsymbol{h}_{s}(\boldsymbol{x}(k))+\boldsymbol{e}_{s} \\
\boldsymbol{z}_{p}(k) & =\boldsymbol{h}_{p}(\boldsymbol{x}(k))+\boldsymbol{e}_{p} \\
\overline{\boldsymbol{z}}_{s}(k / k-1) & =\boldsymbol{h}_{s}(\boldsymbol{x}(k))+\overline{\boldsymbol{e}}_{s}
\end{aligned}
$$

where $k-1$ and $k$ denote two successive discrete times separated by $T_{r}$ seconds, $\boldsymbol{x}(k)$ is the state vector to be estimated at time $k, \boldsymbol{z}_{s}(k)$ (resp. $\boldsymbol{z}_{p}(k)$ ) are the vectors of SCADA (resp. PMU) measurements available at time $k, \boldsymbol{h}_{s}(\cdot)\left(\right.$ resp. $\left.\boldsymbol{h}_{p}(\cdot)\right)$ are functions relating SCADA (resp. PMU) measurements with the state vector, $\boldsymbol{D}_{k}$ is a matrix built from an $m \times m$ identity matrix by removing the rows corresponding to missing measurements at time $k, \overline{\boldsymbol{z}}_{s}(k / k-1)$ is the vector of predicted SCADA measurements available at time $k$ and relying on using previous TSEs, and $\boldsymbol{e}_{s}, \boldsymbol{e}_{p}$ and $\overline{\boldsymbol{e}}_{s}$ are unknown vectors of measurement and prediction errors.

\section{B. Correction step}

The correction step yields the estimate of the state vector $\hat{x}(k / k)$ at time $k$, by processing the predicted SCADA measurements $\overline{\boldsymbol{z}}_{s}(k / k-1)$, the latest available synchronized phasor measurements $\boldsymbol{z}_{p}(k)$ and the SCADA measurements $\boldsymbol{z}_{s}(k)$ received in the interval $[k-1, k]$.

Assuming Gaussian error distributions, the maximum likelihood estimation of state vector $\boldsymbol{x}(k / k)$ is found by solving the following optimization problem:

$$
\min _{\boldsymbol{x}(k / k)} J(\boldsymbol{x}(k / k))
$$

where the objective function is detailed in Eq. (5). In that equation, $\boldsymbol{R}, \boldsymbol{U}$ and $\boldsymbol{M}$ are covariance matrices. They are taken as diagonal matrices, for simplicity, with entries being the variance associated to the various noises in Eqs. (1, 2, 3).

A rectangular coordinate formulation is used, i.e. the state vector $\boldsymbol{x}$ is composed of real and imaginary parts of complex bus voltages. This leads to linear equations (2).

In addition zero injections are treated as linear equality constraints for higher accuracy.

The resulting constrained optimization problem is solved by Hachtel's augmented matrix method, for its higher numerical robustness [1]. The iterations are initialized with the state vector $\hat{\boldsymbol{x}}(k-1 / k-1)$ computed at the previous time step $k-1$.

\section{SCADA measurement prediction step}

This step consists of predicting the near-future values of SCADA measurements using a time series analysis method. Note that, in each TSE, all SCADA measurements are forecasted even if a SCADA measurement is available, which is an improvement with respect to the previous work in [3].

A prediction relying on data available at the current time $k$ and last time $k-1$ can be written in general compact form as:

$$
\overline{\boldsymbol{z}}_{s}(k+1 / k)=\boldsymbol{\phi}\left[\boldsymbol{h}_{s}(\hat{\boldsymbol{x}}(k / k)), \overline{\boldsymbol{z}}_{s}(k / k-1)\right]
$$

Regarding the predictor $\phi(\cdot)$, among various techniques available in the literature [9], Single Exponential Smoothing and Holt's Linear (HL) methods were tested. The latter was found slightly more accurate, and is considered in this paper. It was used in [10], but for prediction of the state variables (instead of SCADA measurements). This method was designed to handle trends. It consists of the following recursive equations:

$$
\overline{\boldsymbol{z}}_{s}(k+1 / k)=\boldsymbol{F}_{k} \boldsymbol{h}_{s}(\hat{\boldsymbol{x}}(k / k))+\boldsymbol{g}_{k}
$$

where

$$
\begin{aligned}
\boldsymbol{F}_{k} & =\alpha(1+\beta) \boldsymbol{I} \\
\boldsymbol{g}_{k} & =(1+\beta)(1-\alpha) \overline{\boldsymbol{z}}_{s}(k / k-1)-\beta \boldsymbol{a}_{k-1}+(1-\beta) \boldsymbol{b}_{k-1} \\
\boldsymbol{a}_{k} & =\alpha \boldsymbol{h}_{s}(\hat{\boldsymbol{x}}(k / k))+(1-\alpha) \overline{\boldsymbol{z}}_{s}(k / k-1) \\
\boldsymbol{b}_{k} & =\beta\left(\boldsymbol{a}_{k}-\boldsymbol{a}_{k-1}\right)+(1-\beta) \boldsymbol{b}_{k-1}
\end{aligned}
$$




$$
\begin{aligned}
J(\boldsymbol{x}(k / k)) & =\frac{1}{2} \underbrace{\left[\boldsymbol{z}_{s}(k)-\boldsymbol{D}_{k} \boldsymbol{h}_{s}(\boldsymbol{x}(k / k))\right]^{T} \boldsymbol{D}_{k} \boldsymbol{R}^{-1} \boldsymbol{D}_{k}^{T}\left[\boldsymbol{z}_{s}(k)-\boldsymbol{D}_{k} \boldsymbol{h}_{s}(\boldsymbol{x}(k / k))\right]}_{\text {SCADA measurements }} \\
& +\frac{1}{2} \underbrace{\left[\boldsymbol{z}_{p}(k)-\boldsymbol{h}_{p}(\boldsymbol{x}(k / k))\right]^{T} \boldsymbol{U}^{-1}\left[\boldsymbol{z}_{p}(k)-\boldsymbol{h}_{p}(\boldsymbol{x}(k / k))\right]}_{\text {PMU measurements }} \\
& +\frac{1}{2} \underbrace{\left[\overline{\boldsymbol{z}}_{s}(k / k-1)-\boldsymbol{h}_{s}(\boldsymbol{x}(k / k))\right]^{T} \boldsymbol{M}^{-1}\left[\overline{\boldsymbol{z}}_{s}(k / k-1)-\boldsymbol{h}_{s}(\boldsymbol{x}(k / k))\right]}_{\text {predicted SCADA measurements }}
\end{aligned}
$$

$\boldsymbol{F}_{k}$ is an estimate of the series "level" and $\boldsymbol{g}_{k}$ of its slope, both at time $k . \boldsymbol{I}$ is a unit matrix.

\section{Analysis of innovation vector}

The innovation vector, i.e. the difference between predicted and measured values:

$$
\boldsymbol{v}(k)=\boldsymbol{z}_{s}(k)-\overline{\boldsymbol{z}}_{s}(k / k-1)
$$

can be used in order to distinguish between gross errors in measurements and a significant change in the operating state [5], [10]. This analysis can be extended to synchronized phasor measurements.

\section{E. Analogy with (extended) Kalman filter}

Figure 2 shows that, with its prediction, innovation analysis and correction steps, the proposed method offers some similarity with an (extended) Kalman filter [10]. However, in the proposed method the prediction is made on the measurement vector in order to avoid using a transition model for the bus voltages, which is difficult to obtain in practice.

\section{Simulation Results}

\section{A. Test system and scenarios}

The performance of the proposed TSE was tested on the Nordic system shown in Fig. 3 and documented in [11]. Detailed time simulations were performed to obtain the system evolution over a few minutes following a severe disturbance.

A three-phase fault was applied on line 4032-4044 and cleared in five cycles by opening the line. Two scenarios were considered. The first one does not include remedial actions, and the system experiences long-term voltage instability under the effect of load tap changers and overexcitation limiters. The second scenario is identical up to the point where undervoltage load shedding takes place, which stabilizes the system. This curtailment is performed in several steps, with $100 \mathrm{MW}$ shed at bus 1041 and $200 \mathrm{MW}$ at bus 1044 .

Based on the "exact" system evolution provided by time simulation, "exact" values of the measured quantities were computed and processed to add time delays in accordance with typical gathering of SCADA measurements. Finally, Gaussian distributed measurement noises were added to obtain "measured" values.

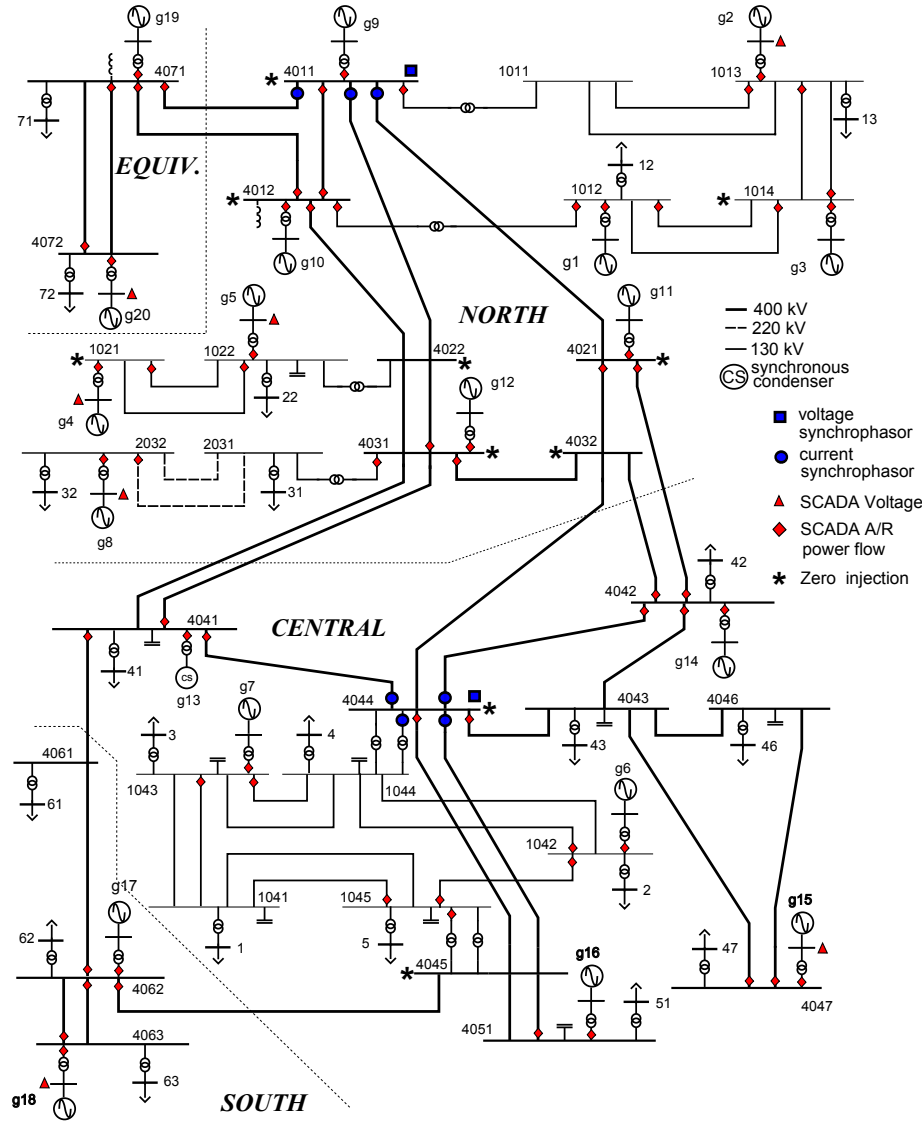

Figure 3. Nordic system (including distribution buses) with its measurement configuration

It was considered that SCADA measurements were transmitted every $T_{i}$ seconds to the control center with a transmission delay $\tau_{i} . T_{i}$ and $\tau_{i}$ vary from one bus to another, with $2 \leq T_{i} \leq 5 \mathrm{~s}$ and $0.1 \leq \tau_{i} \leq 0.5 \mathrm{~s}$. At a given bus, measurements are collected at different instants, leading to a delay $\delta_{j i}$ in the range $[0.1,0.9] \mathrm{s}$, different from one measurement to another. SCADA measurements involve 7 voltage magnitudes (at generator buses) and 66 pairs of active/reactive power flows, for a total of $m=139$ measurements.

Two (multi-channel) PMUs were considered: one at bus 4044 , providing one voltage and four current synchrophasors and one at bus 4011, providing one voltage and three currents.

Finally, ten pairs of zero injections (at buses 4011, 4012, 1014, 1021, 4022, 4021, 4031, 4032, 4044 and 4045) were 
taken into account as constraints.

The measurement configuration is shown in detail in Fig. 3 .

Transformers between transmission and distribution were not included (although they were present in the dynamic simulation, experiencing numerous tap changes). This led to a TSE involving 32 transmission buses, 20 generator buses and 80 line/transformer branches.

The period of TSE execution $T_{r}$ was set to 0.5 second.

Network topology is updated in the TSE execution that follows the line tripping. On the other hand, the network state was tracked without knowing about the overexcitation limitations, the load tap changes and the load curtailments.

\section{B. Measurement standard deviations}

The accuracy of SCADA power flow measurements was set to $0.5 \%$ of full scale power, which was itself set to 1.5 times the nominal power $S_{n o m}$. This leads to a standard deviation:

$$
\sigma_{\text {scada }}=\frac{0.0051 .5 S_{\text {nom }}}{3 S_{\text {base }}} \text { in per unit }
$$

PMU current measurements were assumed five times more accurate than SCADA power flow measurements:

$$
\sigma_{p m u}=\frac{\sigma_{s c a d a}}{5} \text { in per unit }
$$

Finally, predicted SCADA measurements were assumed to be $K$ times less accurate than SCADA measurements:

$$
\sigma_{\text {pred }}=K \sigma_{\text {scada }}
$$

\section{Accuracy indices and initial tuning of the TSE}

In order to assess the overall accuracy of the TSE over the whole simulation, two indices were considered:

- the Mean Absolute Percentage Error (MAPE) evaluates the voltage magnitude mean absolute error in percent:

$$
M A P E=\frac{1}{T} \sum_{k=1}^{T} \frac{1}{N}\left(\sum_{i=1}^{N} \frac{\left|V_{i}^{e x}(k)-V_{i}^{e s t}(k)\right|}{V_{i}^{e x}(k)} 100 \%\right)
$$

where $k$ denotes the discrete time, the upperscript $e x$ (resp. est) refers to exact (resp. estimated) values, $T$ is the total number of TSE executions and $N$ the number of buses;

- the Mean Absolute Error (MAE) evaluates the accuracy of voltage phase angles (in degrees):

$$
M A E=\frac{1}{T} \sum_{k=1}^{T} \frac{1}{N}\left(\sum_{i=1}^{N}\left|\theta_{i}^{e x}(k)-\theta_{i}^{e s t}(k)\right|\right)
$$

First, the factor $K$ in 15 was tuned. The voltage collapse case was used to this purpose, as it involves larger deviations of the system operation point. In this determination, the prediction consisted of merely taking the last estimated value of a SCADA measurement as prediction for the next TSE run, as in [3]. $K$ was varied over a wide range and the value leading to the smallest MAPE index, i.e. $K=3.1$, was selected in all subsequent simulations.

Next, the $\alpha$ and $\beta$ parameters in (7) 8) were varied and the combination leading to the smallest value of the MAPE
Table I

MAPE AND MAE INDICES

\begin{tabular}{rcc}
\hline \hline & $M A P E(\%)$ & $M A E$ (degrees) \\
\hline No disturbance & 0.057854 & 0.036848 \\
Voltage instability & 0.121980 & 0.063582 \\
Load shedding & 0.082208 & 0.044838 \\
\hline
\end{tabular}

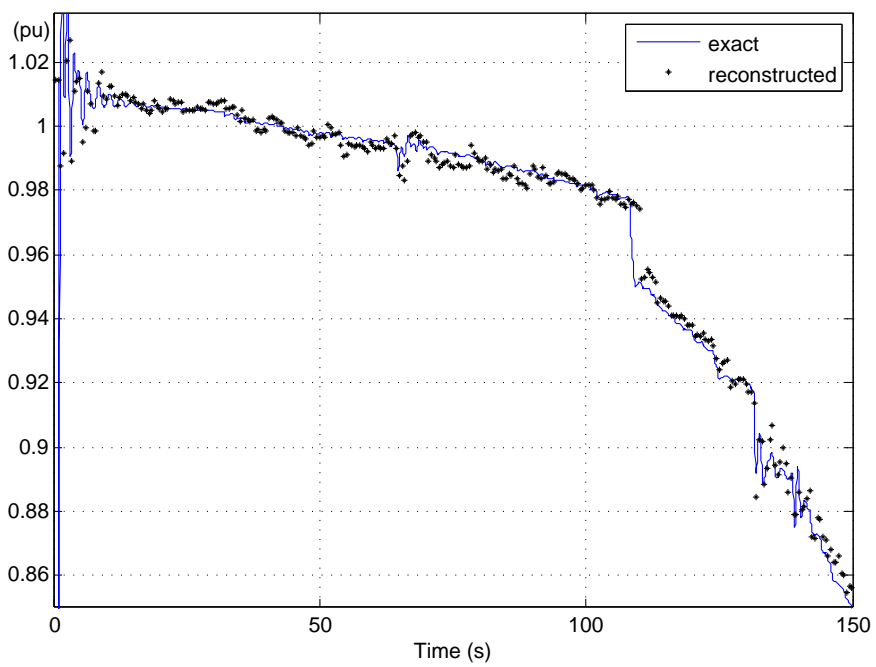

Figure 4. Exact and tracked voltage magnitude at bus 1042; voltage collapse

index was identified as $\alpha=0.6$ and $\beta=0.5$, also used in all subsequent simulations.

The values of the MAPE and MAE indices are given in Table I] for the aforementioned two scenarios as well as when the system is in steady-state. The latter case is provided as reference, since the TSE errors result from measurement noise only (no transient, no time skew). Note that the first ten seconds after fault inception were not included in the calculation of the indices, since the tracked states are not expected to be accurate immediately after a fault.

\section{Example of system evolution tracking}

Figures 4 and 5 show (with solid blue line) the exact and (with black dots) the tracked evolutions of the voltage magnitudes at two buses, most affected by long-term voltage instability and undervoltage load shedding. In the unstable case of Fig. 4, the final voltage collapse (due to loss of synchronism of g6) is not shown, to preserve legibility.

It can be seen that the TSE restitutes the overall system evolution. A closer look at Fig. 4, near $t=110 \mathrm{~s}$, confirms that after a sudden change in the system (in this case, an important generator switching under field current limit), the tracked evolution follows the exact one with a delay, due to the non synchronized SCADA measurements, and the absence of PMUs near the buses of concern. This delay, however, is rather short.

\section{E. Detailed analysis of TSE accuracy}

In order to characterize the accuracy of estimates provided by the proposed TSE, Monte Carlo simulations were per- 


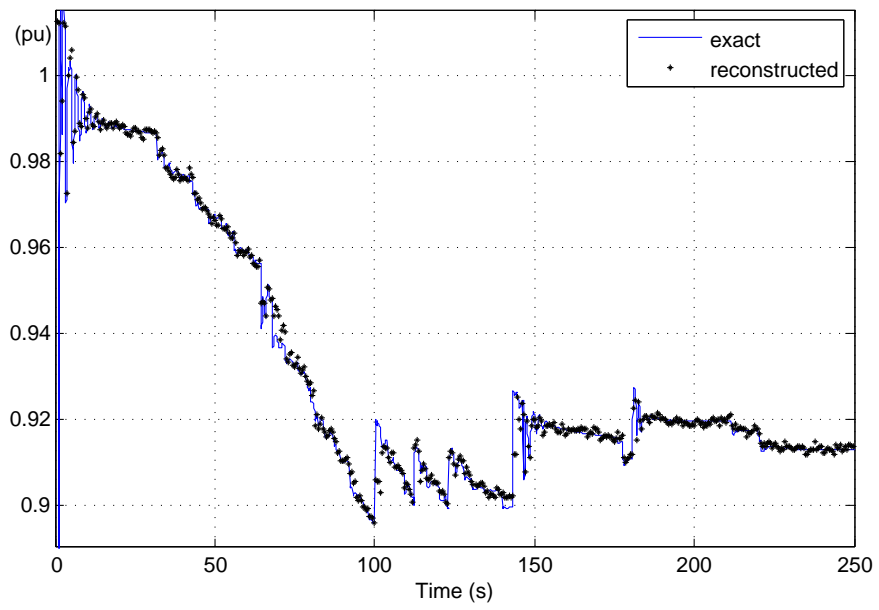

Figure 5. Exact and tracked voltage magnitude at bus 1041; load shedding

formed and the results assessed as follows. A set of $s=500$ simulations was built, differing by the random noise applied to measurements, as well as by the transmission delay $\tau_{i}$ randomly chosen in the interval $[0.1,0.5] \mathrm{s}$. For each voltage magnitude and power flow provided with a SCADA measurement, the mean and standard deviation of the corresponding $s$ estimates was computed, every $T_{r}=0.5 \mathrm{~s}$, and for each of three scenarios listed in Table I]

Thus, the average and standard deviation were computed as:

$$
\begin{aligned}
& \mu_{j}(k)=\frac{1}{s} \sum_{i=1}^{s} e_{j, i}(k) \\
& \sigma_{j}(k)=\sqrt{\frac{1}{s} \sum_{i=1}^{s}\left(e_{j, i}(k)-\mu_{j}(k)\right)^{2}}
\end{aligned}
$$

where

$$
e_{j, i}(k)=h_{j}\left(\hat{\boldsymbol{x}}_{i}(k / k)\right)-h_{j}(\boldsymbol{x}(k))
$$

is the estimation error of the $j$-th measured quantity, at the $k$-th discrete time $(k=1, \ldots, T)$, provided by the $i$-th simulation $(i=1, \ldots, s)$.

Finally, for each measured quantity, a global mean and standard deviation were computed over all $T$ discrete times:

$$
\begin{aligned}
& \bar{\mu}_{j}=\frac{1}{T} \sum_{k=1}^{T} \mu_{j}(k) \\
& \bar{\sigma}_{j}=\sqrt{\frac{1}{T} \sum_{k=1}^{T} \sigma_{j}(k)^{2}}
\end{aligned}
$$

For a sample of three active power flows, Table III shows respectively the standard deviation of the measurement noise, given by [13, and the standard deviation of the estimation error, given by 222 , in each of the three cases already considered in Table I. The values of $\bar{\sigma}_{j}$ being smaller than those of $\sigma_{j}$ shows the filtering capability of the proposed TSE in spite of the significant system transients.

\section{CONClusion}

A new tracking state estimation has been proposed based on the simultaneous processing of asynchronously gathered
Table II

STANDARD DEVIATIONS (IN PER UNIT) OF MEASUREMENT NOISE AND ESTIMATION ERRORS FOR THREE MEASURED ACTIVE POWER FLOWS

\begin{tabular}{c|c|ccc}
\hline \hline \multirow{2}{*}{ line } & $\sigma_{j}$ of & \multicolumn{3}{|c}{$\bar{\sigma}_{j}$ of estimation error } \\
& measurement & no & load & voltage \\
noise & disturbance & shedding & instability \\
\hline $4047-4043$ & 0.03500 & 0.031725 & 0.0289680 & 0.032219 \\
$2032-2031$ & 0.01250 & 0.0095313 & 0.0120020 & 0.010977 \\
$1043-1041$ & 0.00875 & 0.0077606 & 0.0068832 & 0.007706 \\
\hline \hline
\end{tabular}

SCADA measurements and a limited number of synchrophasor measurements. SCADA measurement prediction is used to ensure network observability.

The results show the ability to track network state evolution after a major disturbance. In particular sudden system changes are tracked correctly, although with a short delay due to the non-synchronized SCADA measurements. Furthermore, Monte-Carlo simulations confirm the ability to filter measurement noise.

While simple, the method bridges the gap between standard static and full dynamic state estimations. It is not expected to be run more often than - say - every tenth of a second. Hence, to deal with short-term angle or voltage dynamics, a richer PMU configuration and a true dynamic state estimator are needed. However, for a wide range of slower phenomena monitored from a control center, such as long-term voltage instability, slow interarea oscillations or thermal overloads, the method can improve situational awareness when a limited number of PMUs is available.

Bad data analysis and pseudo-measurement covariance determination are among the aspects further investigated.

\section{REFERENCES}

[1] A. Abur and A. G. Exposito, Power system state estimation: theory and implementation. New York: Marcel Dekker, 2004.

[2] A. G. Phadke and J. S. Thorp, Synchronized Phasor Measurements and Their Application. New York: Springer, 2008.

[3] M. Glavic and T. Van Cutsem, "Tracking network state from combined scada and synchronized phasor measurements," in Bulk Power Systems Dynamics and Control-IX (IREP), Rethymnon (Greece), Aug. 2013. [Online]. Available: IEEEXplore,DOI:10.1109/IREP.2013.6629376

[4] R. Masiello and F. C. Schweppe, "A tracking state estimator," IEEE Trans. Power App. and Syst, vol. PAS-90, pp. 1025-1033, Mar. 1971.

[5] D. M. Falcao, P. A. Cooke, and A. Brameller, "Power system tracking state estimation and bad data processing," IEEE Trans. Power App. Syst., vol. PAS-101, pp. 325-333, Feb. 1982.

[6] H. Singh and F. L. Alvarado, "Fast approximations to LAV solutions for state estimation of power systems," in Proc. 11th Power System Computation Conference (PSCC), Avignon, France, Aug. 1993.

[7] "IEEE standard c37.118.1-2011: IEEE standard for synchrophasors for power systems," Tech. Rep., 2011.

[8] M. Gol and A. Abur, "A hybrid state estimator for systems with limited number of PMUs," IEEE Trans. Power Syst., vol. 29, pp. 1-7, Aug. 2014.

[9] S. Makridakis and S. Wheelwright, Forecasting methods and applications. John Wiley and Sons, 1978.

[10] A. M. L. da Silva, M. B. D. C. Filho, and J. F. de Queiroz, "State forecasting in electric power systems," Proc. IEE on Gener, Transm. and Distrib., vol. 30, pp. 237-244, Sep. 1983.

[11] T. Van Cutsem and L. Papangelis, "Description, modeling and simulation results of a test system for voltage stability analysis (version 6)," University of Liège, Belgium, Tech. Rep., Nov. 2013. 